\title{
The importance of beta cell characterisation: generating human beta cells by differentiating human embryonic stem cells
}

\author{
G. S. Korbutt • T. Y. Yeung • C. E. Ellis
}

Received: 14 October 2011 /Accepted: 7 November 2011/Published online: 7 December 2011

(C) Springer-Verlag 2011

Keywords Beta cells $\cdot$ Embryonic stem cells

\author{
Abbreviations \\ ARX Aristaless related homeobox \\ BRN4 Brain-specific homeobox/POU domain protein 4 \\ hESC Human embryonic stem cell \\ MMP2 Matrix metallopeptidase 2
}

Exogenous insulin administration continues to be the most common means of correcting the absolute insulin deficiency in patients with type 1 diabetes mellitus. Nevertheless, the best available insulin therapy does not control blood glucose levels in a physiological manner, leading to several serious metabolic and neurovascular complications [1]. Beta cell replacement offers an attractive alternative, potentially enabling precise glycaemic control. Clinical islet transplantation, for instance, has proven effective in improving glucose homeostasis, reducing episodes of hypoglycaemia and achieving insulin independence [2]. However, the shortage of donor islet tissues is a major restriction for widespread application of beta cell replacement therapies. The recent developments in stem cell research suggest that human embryonic stem cells (hESCs)

G. S. Korbutt • T. Y. Yeung • C. E. Ellis

Department of Surgery, Alberta Diabetes Institute,

Edmonton, AB, Canada

G. S. Korbutt $(\varangle) \cdot$ T. Y. Yeung $\cdot$ C. E. Ellis

5-002 Li Ka Shing Centre for Health Research Innovation, University of Alberta,

Edmonton, AB, Canada T6G 2E1

e-mail: korbutt@ualberta.ca hold great promise for fulfilling this need for an unlimited supply of readily available insulin-producing cells.

Embryonic stem cells are pluripotent cells derived from the inner cell mass of blastocysts. They exhibit the capacity to develop into the three embryonic germ layers (endoderm, mesoderm and ectoderm). To generate beta cells from hESCs, current approaches have been guided by knowledge of pancreatic development. By recapitulating these developmental signals, several groups have been able to differentiate hESCs into insulinpositive cells [3-6]. Jiang et al. utilised a differentiation protocol omitting serum from the culture medium and reported the presence of islet-like clusters that secreted Cpeptide in response to glucose [3]. However, C-peptidepositive cells only accounted for $2-8 \%$ of all cells in the islet-like cluster [3]. When transplanted into chemically induced diabetic mice, the islet-like clusters prolonged survival and secreted C-peptide in response to a glucose challenge [4]. Novocell (now known as ViaCyte, San Diego, CA, USA) developed another differentiation protocol in which hESC-derived insulin positive cells could secrete C-peptide but only in the presence of secretagogues like $\mathrm{KCl}$ [5]. When hESC-derived pancreatic progenitors were transplanted into athymic mice, they reported that these cells could also produce human insulin and C-peptide levels comparable to 3,000 human islets [6]. While the results reported by the ViaCyte group were impressive, Matveyenko et al. had difficulty replicating these results in athymic rats [7]. In addition, the recipient mice responded poorly to glucose tolerance testing, which suggests that insulin secretion was not glucose dependent. Clearly, improvements to directed differentiation attempts are necessary to overcome two significant hurdles that 
hinder the field of ESC differentiation of human beta cells: (1) developing a highly efficient beta cell differentiation protocol that enables the production of therapeutic quantities of functionally mature beta cells; and (2) achieving glucose-dependent insulin secretion in vitro and in vivo from differentiated insulin-positive cells.

In a study reported in this issue of Diabetologia, Basford et al. utilised a new reporter hESC line $\left(I N S^{G F P / w}\right)$ differentiated over 22 days using the 'Nostro protocol' and HES2 cells differentiated over the same period [8]. The $I N S^{G F P / w}$ line efficiently generates insulin-producing cells, supporting the use of the protocol to produce hESC-derived insulin-positive cells that were characterised extensively using microarray, quantitative PCR, secretions assay, electron microscopy and electrophysiology and compared with the hESC-derived insulin-positive line for features of adult beta cells. The hESC-derived insulin-positive cells were stained for insulin, glucagon, somatostatin, green fluorescent protein (GFP), brain-specific homeobox/POU domain protein 4 (BRN4), aristaless related homeobox (ARX), C-peptide, GLUT-2, cytokeratin 19 (CK19) and matrix metallopeptidase 2 (MMP2), an impressive list that explores the maturity of the cells. As expected, the $I N S^{G F P / w}$ cells had complete co-localisation of insulin and GFP. The hESC-derived insulin-positive cells produced both C-peptide and GLUT-2, and were frequently polyhormonal. They also produced both CK19 and MMP2, markers of immature beta cells, suggesting an immature endocrine phenotype. Additionally, these cells stained positively for the alpha cell transcription factors ARX and BRN4. Despite differences between adult beta cells and the hESC-derived insulin-positive cell line, there are many similarities, including the production of insulin-like granules, ion channel activity and intracellular responsiveness, and therefore this cell line may potentially generate fully functioning beta cells.

Molecular analysis of the hESC-derived insulin-positive cells and adult human beta cells revealed key differences in global gene expression, such as the increased expression of a cluster of transcription factors important in endocrine cell development compared with human islets, and significantly lower INS, GCG and SST expression; however, there were also many important similarities, particularly in genes related to endocrine cell development and function.

Electron microscopy was used to examine the cellular ultrastructure of the hESC-derived insulin-positive cells compared with that of murine beta cells. The hESC-derived insulin-positive cells contained fewer total granules with atypical morphology compared with the murine beta cells; however, the hESC-derived insulin-positive cells had numerous mitochondria, which is typical for an islet cell phenotype. Immuno-gold labelling of the INS: $\mathrm{GFP}^{+}$cells confirmed defined insulin and glucagon granules and cytosolic insulin and glucagon. Basford et al. concluded that the hESCderived insulin-positive cells were polyhormonal, with granules for insulin and glucagon, but granule levels were significantly lower than those in the adult murine islets.

Although the hESC-derived insulin-positive cells did not secrete insulin at levels comparable to adult beta cells in response to glucose, they consistently released insulin under direct cellular repolarisation at similar amounts of insulin per insulin-positive cell. There were no differences in GLUT-1 levels between the INS: $\mathrm{GFP}^{+}$cells and human beta cells. $\mathrm{K}_{\mathrm{ATP}}$ channels were found to be more highly expressed in the INS: $\mathrm{GFP}^{+}$cells than in human islets, but the channels were heterogeneously active; $\mathrm{K}_{\text {ATP }}$ and voltage-gated $\mathrm{Ca}^{2+}$ channel activity of insulin-positive cells was variable and was not similar to that observed in mature beta cells. Together, these results imply that, although the hESC-derived insulin-positive cells are not fully functional, they resemble immature endocrine cells with sufficient glucose-sensing and metabolism gene expression and some normal channel activity.

This study by Basford et al. represents the first comprehensive attempt to characterise hESC-derived insulin-positive cells at the molecular and functional level. The Wheeler group utilised an impressive and novel protocol described by Nostro, which yielded differentiated cells that were up to $25 \%$ C-peptide positive [9]. By examining the maturation of ESCs by gene expression, ultrastructural features and electrophysiological characteristics, Basford et al. have elucidated potential targets for improving the beta cell differentiation protocol; for example, the authors suggest that the addition of certain key factors, Mafa and $N k x 6.1$, may improve the function and yield of hESC-derived human beta cells. Overall, the iterative approach to developing differentiation protocols may be better guided by correlating functional and molecular data. Thus, this approach serves to inform future attempts at directed differentiation. Although Basford et al. did not observe maturation of the transplanted INS: $\mathrm{GFP}^{+}$cells into functional beta cells, possibly due to beta cell death or transdifferentiation of beta cells to alpha cells in vivo, the full characterisation of differentiated hESCs is an important and exciting step towards generating true human beta cells. Finally, when evaluating the outcome of beta cell replacement studies there are also safety concerns, in that inappropriate insulin release, triggered from non-physiological stimuli, should not take place. Gene expression studies could therefore also consider genes that should be physiologically repressed in mature beta cells, such as low $K_{\mathrm{m}}$ hexokinases [10] and the lactate/pyruvate transporter monocarboxylate transporter 1 (MCT1) [11, 12].

Acknowledgements Research in the authors' departments has been supported from grants from the Juvenile Diabetes Research Founda- 
tion, the Canadian Institute of Health Research, the Canadian Diabetes Association, the Alberta Heritage for Medical Research Foundation and the Alberta Diabetes Research Institute.

Contribution statement All authors were responsible for the conception and design of the manuscript, drafting the article and revising it critically for important intellectual content. All authors approved the version to be published.

Duality of interest The authors declare that there is no duality of interest with this manuscript.

\section{References}

1. The Diabetes Control and Complications Trial Research Group (1993) The effect of intensive treatment of diabetes on the development and progression of long-term complications in insulin dependent diabetes mellitus. N Engl J Med 329:977-986

2. Shapiro AM, Lakey JR, Ryan EA et al (2000) Islet transplantation in seven patients with type 1 diabetes mellitus using a glucocorticoidfree immunosuppressive regimen. N Engl J Med 343:230-238

3. Jiang J, Au M, Lu K et al (2007) Generation of insulin-producing islet-like clusters from human embryonic stem cells. Stem Cells 25:1940-1953

4. Eshpeter A, Jiang J, Au M et al (2008) In vivo characterization of transplanted human embryonic stem cell-derived pancreatic endocrine islet cells. Cell Prolif 41:843-858
5. D'Amour KA, Bang AG, Eliazer S et al (2006) Production of pancreatic hormone-expressing endocrine cells from human embryonic stem cells. Nat Biotechnol 24:1392-1401

6. Kroon E, Martinson LA, Kuniko K et al (2008) Pancreatic endoderm derived from human embryonic stem cells generates glucose-responsive insulin-secreting cells in vivo. Nat Biotechnol 26:443-542

7. Matveyenko AV, Georgia S, Bhushan A, Butler PC (2010) Inconsistent formation and nonfunction of insulin-positive cells from pancreatic endoderm derived from human embryonic stem cells in athymic nude rats. Am J Physiol Endocrinol Metab 299: E713-E720

8. Basford CL, Prentice KJ, Hardy AB et al (2012) The functional and molecular characterisation of human embryonic stem cell-derived insulin-positive cells compared with adult pancreatic beta cells. Diabetologia. doi:10.1007/s00125-0112335-x

9. Nostro MC, Sarangi F, Ogawa S et al (2011) Stage-specific signaling through TGF $\beta$ family members and WNT regulates patterning and pancreatic specification of human pluripotent stem cells. Development 138:861-871

10. Schuit F, Moens K, Heimberg H, Pipeleers D (1999) Cellular origin of hexokinase in pancreatic islets. J Biol Chem 274:3280332809

11. Otonkoski T, Jiao H, Kaminen-Ahola N et al (2007) Physical exercise-induced hypoglycemia caused by failed silencing of monocarboxylate transporter 1 in pancreatic beta cells. Am J Human Genet 81:467-474

12. Thorrez L, Laudadio I, Van Deun K et al (2011) Tissue-specific disallowance of housekeeping genes: the other face of cell differentiation. Genome Res 21:95-105 